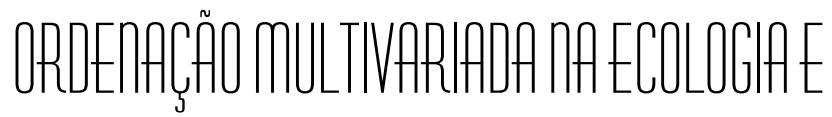

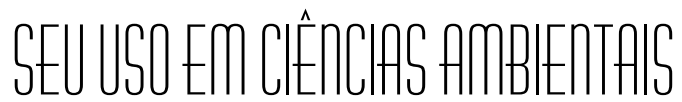 \\ PAULO INÁCIO PRADO* \\ THOMAS MICHAEL LEWINSOHN** \\ ROBERTO LUIZ DO CARMO**** \\ DANIEL JOSEPH HOGAN****
}

\section{INTRODUÇÃO}

Inventários bióticos tradicionalmente resultam em matrizes onde se registram a presença ou abundância de cada espécie em um dado local ou ambiente. Como estas matrizes podem facilmente ultrapassar várias dezenas de espécies e algumas dezenas de locais, a visualização dos padrões de ocorrência e co-ocorrência das espécies pelos ambientes ou locais é uma tarefa difícil. Em muitas ocasiões dispõe-se, ainda, de variáveis ambientais dos locais, e assim deseja-se, também, correlacionar as distribuições das espécies com características do meio.

Para explorar estes padrões de uma maneira analítica e quantitativa, foram criados na ecologia (ou adaptados de outras áreas de conhecimento) muitos procedimentos estatísticos exploratórios, conhecidos em seu conjunto como análises multivariadas (GAUCH, 1982; PIELOU, 1984; MANLY, 1994; JONGMAN et al., 1995). As análises multivariadas objetivam reduzir um grande número de variáveis a poucas dimensões com o mínimo de perda de informação, permitindo a detecção dos principais padrões de similaridade, de associação e de correlação entre as variáveis.

Estes métodos podem ser usados para qualquer conjunto de objetos ou entidades das quais se tenham tomado várias medidas, e, de fato, alguns deles foram criados ou desenvolvidos no âmbito de outras ciências, como taxonomia, antropologia física, sociologia e psicologia (SNEATH \& SOKAL, 1973; DILLON \& GOLDSTEIN, 1984; MANLY 1994). A despeito do uso de análises multivariadas em diversas áreas de conhecimento, há poucas iniciativas para padronizá-las, e, muitas vezes, os mesmos procedimentos recebem designações diferentes meramente por razões históricas e devido à falta de diálogo interdisciplinar (e.g. DILLON \& GOLDSTEIN, 1984; BERNSTEIN et al. 1988; MANLY, 1994). Além disto, embora os procedimentos estatísticos sejam os mesmos, há diferenças importantes nos protocolos de análise e nas questões enfatizadas, e raramente uma única área de conhecimento esgota todo o potencial das análises multivariadas que utiliza. 
Neste trabalho, experimentamos análises multivariadas de ordenação, que têm tido muito uso em ecologia, em dados ambientais de outras áreas de conhecimento. Nosso objetivo não é nos contrapor a outros tipos de análises, qualitativas ou quantitativas. Antes, pretendemos apontar que muitos dos dados sócio-ambientais prestam-se a este tipo de análise, cabendo aos pesquisadores avaliar se há algum ganho com isto. Pretendemos, também, apresentar o modo como ecólogos abordam e analisam dados, na expectativa de que parte deste instrumental possa ser útil a nossos colegas de outras áreas. Embora os métodos e abordagens de cada área de conhecimento atendam às idiossincrasias de seu paradigma, parte deles pode ser proveitosamente adaptada para uso em outras áreas. Esperamos, assim, estar contribuindo para a construção de metodologias analíticas e quantitativas que viabilizem o trabalho multidisciplinar.

Este trabalho privilegia os aspectos metodológicos. As interpretações das análises não se pretendem exaustivas e seu intuito é indicar algumas das possibilidades abertas. Há um pequeno resumo teórico das análises de ordenação, e, em seguida, a análise de um conjunto de dados, resultantes de uma pesquisa interdisciplinar em meio ambiente, realizada na região de Campinas (HOGAN et al. 1997). Escolhemos este conjunto por representar muito bem o tipo de dados produzido pelas ciências ambientais, tanto em sua complexidade, quanto em sua adequação para análises multivariadas.

\section{MÉTODOS DE ORDENAÇÃO}

Ordenação multivariada é qualquer processo que forneça um pequeno número de variáveis que descrevam as relações entre objetos, a partir de medidas tomadas desses objetos (GAUCH, 1982; PIELOU, 1984; KREBS, 1989; MANLY, 1994; JONGMAN et al. 1995). Esse processo pode ser feito a partir das próprias medidas, ou de uma matriz de distâncias ou de similaridades entre os objetos. O objetivo da ordenação é sumariar um grande número de medidas em poucas variáveis sintéticas, facilitando a identificação de padrões. Na maioria das técnicas de ordenação, as variáveis sintéticas, também denominadas eixos ou componentes, são combinações lineares das medidas originais, do tipo:

$$
Y=a_{1} x_{1}+a_{2} x_{2}+a_{3} x_{3}+\ldots a_{n} x_{n}
$$

onde $\mathrm{x}_{\mathrm{i}}$ são as medidas, e $\mathrm{a}_{\mathrm{i}}$ os seus coeficientes lineares, que expressam sua importância para o eixo (PIELOU, 1984; DILLON \& GOLDSTEIN, 1984; BERNSTEIN et al,. 1988; MANLY, 1994; JONGMAN et al. 1995). A participação de cada medida em um eixo também pode ser medida pelo seu peso ("loading"), que é a sua correlação com o eixo.

A maneira como os eixos são calculados é que define cada técnica de ordenação, mas as mais usadas em ciências naturais obedecem a duas restrições importantes. A primeira é que os eixos não são correlacionados, e, assim, cada eixo 
captura tendências independentes dos dados. Alémdisso, os eixos possuem uma ordem de importância: o primeiro é aquele que explica a maior parcela da variação dos dados, e o segundo, o que explica a segunda maior parte da variação, e assim por diante (PIELOU, 1984; DILLON \& GOLDSTEIN, 1984; BERNSTEIN et al. 1988; MANLY, 1994; JONGMAN et al. 1995). Em muitos casos, os dois ou três primeiros eixos explicam grande parte da variação (60 a 90\%), o que permite usá-los para descrever todo o sistema sem grande perda de informação. A coordenada de cada objeto ao longo de um eixo, ou escore, é dado por Y (fórmula acima), o que torna possível usar os eixos em um plano cartesiano para representar relações multivariadas em poucas dimensões (KREBS 1989, MANLY 1994).

A análise de componentes principais (PCA) é indicada para conjuntos de medidas correlacionadas linearmente, o que permite reduzi-las a poucas variáveis sintéticas, denominadas componentes principais (PIELOU 1984, MANLY 1994). Os coeficientes dos eixos são calculados pelos autovetores da matriz de covariâncias ou de correlações entre as medidas (PIELOU 1984, DILLON \& GOLDSTEIN 1984, MANLY 1994). Os eixos são extraídos de modo que o primeiro explique a maior parte da variância, o segundo explique a maior parte da variância residual, e assim, sucessivamente. Desta maneira, os eixos não são correlacionados, e podem ser interpretados como vetores independentes (DILLON \& GOLDSTEIN, 1984; MANLY, 1994). Quanto maior a correlação entre as medidas originais, maior será a parcela de variação explicada pelos primeiros eixos.

Os coeficientes das variáveis, ou seus pesos, são usados para interpretar os eixos. As variáveis mais importantes são as de maiores coeficientes e pesos, negativos ou positivos. $\mathrm{O}$ sinal dos pesos indica se a correlação entre as medidas representadas pelo eixo é positiva ou negativa. Na interpretação dos eixos é comum atribuir-lhes nomes, que refletem as principais tendências que eles representam. Na biologia, por exemplo, é bem conhecido que um PCA de medidas do corpo de animais resulta em um primeiro eixo, cujos pesos de todas variáveis têm o mesmo sinal e aproximadamente o mesmo valor. Este eixo é tradicionalmente interpretado como o aumento concomitante de todas as medidas do corpo, ou seja, uma expressão do tamanho do animal. Os eixos seguintes, não correlacionados com o primeiro, por definição, são interpretados como variação morfométrica independente do tamanho, ou seja, vetores de mudança de forma do corpo (MOSIMANN \& JAMES, 1979; BOOKSTEIN, 1989; PERES-NETO, 1995).

A análise de coordenadas principais (PCO) é uma generalização do PCA, na qual os autovalores são extraídos de uma matriz de similaridade ou de distâncias (MANLY, 1994; JONGMAN et al., 1995). A principal vantagem é que esta técnica pode ser aplicada quando as relações entre as variáveis não são lineares. Outra vantagem é que não são necessárias as medidas originais, mas apenas a matriz de similaridades, que pode, inclusive, seguir uma escala qualitativa, desde que ordenada (e.g., muito baixo/baixo/médio/alto/muito alto). Os demais pressupostos e modos de interpretação são os mesmos de um PCA. 


\section{UM ESTUDO DE CASO: PADRÕES DEMOGRÁFICOS E QUALIDADE AMBIENTAL NAS BACIAS DO PIRACICABA E CAPIVARI}

Nosso objetivo foi analisar as relações de variáveis demográficas entre si e com variáveis de qualidade ambiental. As variáveis demográficas são, em geral, muito correlacionadas, e possivelmente seguem algumas poucas tendências comuns. Isto permite decompor as correlações entre essas variáveis demográficas em vetores sintéticos, através de técnicas de ordenação. Uma vez definidos tais vetores demográficos sintéticos, é possível analisar suas relações com outras classes de variáveis. Neste trabalho, usamos as correlações entre os eixos da ordenação com duas variáveis ambientaispara exemplificar uma possibilidade de análise das relações entre vetores demográficos e qualidade ambiental,

As variáveis demográficas e ambientais foram obtidas de dados censitários (IBGE) para os 47 municípios paulistas criados antes de 1980 nas Bacias dos Rios Piracicaba e Capivari (BPC, Apêndice). Essas bacias localizam-se, na sua maior extensão, no centro-leste do Estado de São Paulo. As cabeceiras da bacia do rio Piracicaba se encontram nos municípios mineiros de Camanducaia, Extrema, Toledo e Itapeva. Os municípios analisados neste trabalho estão na Região Administrativa de Campinas, e formam uma das áreas mais urbanizadas, populosas e industrializadas do Brasil (HOGAN et al. 1997, 1998).

Com os dados dos Censos Demográficos do IBGE, sistematizados por HOGAN et al. (1998), definimos quatro variáveis demográficas, cujos valores encontram-se no Apêndice:

• População residente no município em 1991. (Variável "Pop", no Apêndice);

- $\quad$ Percentual de população urbana no município em $1991=($ população urbana / população residente)x100. (Variável “\%Urban”);

Crescimento vegetativo percentual no município entre 1980-1991 $=($ crescimento vegetativo no período/ população residente em 1980 )x100. (Variável "\%Cveg”);

Saldo migratório percentual no município entre 1980-1991 = (saldo migratório no período / população residente em 1980)x100. (Variável “\%Smigr").

As variáveis de qualidade ambiental também foram:

Percentual de domicílios com ligação de esgoto no município (Censo Demográfico de 1991, IBGE, sistematizadas por HOGAN et al. 1998; variável “\%Esgot”, no apêndice);

Percentual de área não urbana ocupada por vegetação nativa no município em 1988 (Secretaria do Meio Ambiente - SP 1999; variável “\%Mata”). 
Uma análise exploratória mostrou que as relações entre as variáveis demográficas não são lineares, por isso optamos por um PCO, realizado com a matriz de correlações não paramétricas de Spearman. Os vetores dos coeficientes das variáveis demográficas em cada eixo (autovetores) foram usados para interpretar os eixos. Gráficos das variáveis ambientais dos municípios, em função dos seus escores nos eixos do PCO, foram usados para analisar a correlação entre qualidade ambiental e vetores demográficos, a qual foi medida com coeficiente de correlação de Spearman.

Outros detalhes da metodologia, e uma análise de variáveis adicionais, estão em PRADO \& LEWINSOHN (1998). BALTAR et al. (2000) fornecem análises similares de variáveis de crescimento populacional, infra-estrutura e atividade econômica nas Bacias dos Rios Piracicaba, Capivari e Jundiaí, bem como uma explicação matemática detalhada de análises multivariadas.

\section{RESULTADOS DA ANÁLISE}

Os coeficientes das variáveis demográficas no primeiro eixo do PCO são equivalentes e de mesmo sinal e, assim, este eixo pode ser interpretado como o aumento simultâneo de todas elas (Tabela 1). O primeiro eixo responde por 53\% da variação dos dados, mostrando que o principal padrão demográfico na BPC é que o tamanho populacional está positivamente associado a maiores taxas de urbanização, crescimento vegetativo e migração. Portanto, a ordenação confirmou o principal vetor demográfico da região, que foi o crescimento populacional acelerado, associado à urbanização e industrialização (HOGAN et al., 1997). Os municípios com maiores escores no primeiro eixo são Campinas e Sumaré (Fig. 1, Apêndice), os quais possuem grandes tamanhos populacionais e taxas de urbanização superiores a 95\%. No extremo oposto do eixo, estão os municípios com populações pequenas (menos que 5.000 habitantes em 1991), e com mais da metade da população residindo nas áreas rurais, como Pedra Bela, Corumbataí e Mombuca (Fig.1, Apêndice).

O segundo eixo responde por $24 \%$ da variação dos dados, e é fortemente determinado pela variável de saldo migratório percentual. O coeficiente desta variável no segundo eixo é o dobro do segundo maior coeficiente, que é o da variável de percentual de urbanização (Tabela 1). $\mathrm{O}$ crescimento vegetativo percentual foi a variável com menor influência neste eixo (coeficiente $=0,221$, Tabela 1 ). Os coeficientes das variáveis de tamanho populacional e de taxa de urbanização têm sinal contrário aos das variáveis de saldo migratório e crescimento vegetativo (Tabela 1). Logo, o segundo eixo da PCO pode ser interpretado como um vetor de aumento do saldo migratório percentual independente, ou mesmo em oposição ao tamanho populacional e taxa de urbanização (Tabela 1).

Municípios com escores altos no segundo eixo são aqueles com saldo migratório elevado, sem necessariamente serem populosos, ou seja, pólos de atração com tendência a crescimento rápido. Este é o caso do município de Artur Nogueira, que possui o segundo maior escore no segundo eixo (Fig. 1). Sua população é de tamanho médio para a região (28.035 habitantes em 1991), mas teve um aumento de 55\% 
devido ao saldo migratório entre 1980 e 1991 (Apêndice). Jarinu e Monte Mor são outros municípios que possuem escores elevados no segundo eixo e seguem o mesmo padrão (Fig.1). Há, também, dois municípios populosos que tiveram escores elevados, por possuírem os maiores saldos migratórios percentuais na Bacia: Sumaré (saldo de 94\% entre 1980 e 1991), e Santa Bárbara D’Oeste (saldo de 63\%, ver Apêndice). Em um contexto nacional de queda dos níveis de fecundidade, que sobressaiu a partir da década de 1970, o expressivo crescimento populacional de alguns municípios da região só pode ser explicado pela migração. De fato, a bibliografia que discute a evolução populacional na região (HOGAN et al. 1997,1998; BAENINGER 2000) tem mostrado a importância da migração em municípios como Sumaré e Santa Barbara d'Oeste, que fazem parte do eixo de expansão urbana e conurbação ao longo da Rodovia Anhanguera (CARMO, 2001).

É interessante notar que os dois municípios mais populosos da Bacia, Campinas e Piracicaba, têm escores baixos no segundo eixo (Fig. 1), pois tiveram saldos migratórios pequenos no período (4,6\% e 10,4\%, respectivamente, ver Apêndice). A redução da migração para os grandes centros regionais ("pólos consolidados" e "pólos adensados") e o surgimento de novos pólos de atração em processo de urbanização ("pólos periféricos") é uma tendência demográfica bem conhecida no Estado de São Paulo (CUNHA et al., 2000; BAENINGER, 2000), já notada e analisada na BPC pelos autores HOGAN et al. $(1997,1998)$ e CARMO (2001).

Correlacionando o primeiro eixo do PCO com as variáveis ambientais, podemos analisar como o crescimento populacional afetou a qualidade ambiental na BPC. Há uma correlação significativa entre cobertura percentual de vegetação nativa dos municípios e seus escores no primeiro eixo (Fig. 2), indicando a tendência à redução da vegetação nativa nos municípios com maior número de habitantes e taxas de urbanização. Esta tendência é clara para os municípios com escores extremos (Fig.2). Já nos municípios com escores intermediários, a cobertura vegetal é muito variável (e.g. compare Piracaia com Rio das Pedras na Fig. 2) e a correlação não é tão evidente, sugerindo que a conservação de vegetação natural é possível mesmo com o aumento do povoamento e urbanização.

Há uma forte correlação entre o primeiro eixo da $\mathrm{PCO}$ e o percentual de domicílios ligados à rede de esgoto (Fig. 3), mostrando que municípios maiores e mais urbanizados possuem maior cobertura de saneamento básico. Em análises multivariadas independentes, BALTAR et al. (2000) também encontraram uma forte correlação entre crescimento de variáveis de infra-estrutura e crescimento populacional nas Bacias dos Rios Piracicaba, Capivari e Jundiaí. O gráfico também mostra que alguns municípios desviam-se consideravelmente desse padrão, tendo uma cobertura de saneamento menor do que o esperado para seu tamanho populacional e grau de urbanização (Fig. 3). Entre estas exceções, há casos especialmente críticos como Jarinu, Sumaré e Monte Mor, núcleos em franco desenvolvimento e com menos de $40 \%$ dos domićlios ligados à rede de esgoto em 1991 (Fig. 3, Apêndice). Tal desvio em relação ao padrão de abastecimento de cidades do mesmo porte populacional pode estar relacionado ao fato de que estes municípios apresentaram um crescimento populacional 
muito significativo no período. Todos eles praticamente dobraram o número de habitantes entre 1980 e 1991. Assim, Jarinu passou de 6,2 para 10,8 mil habitantes no período; Monte Mor passou de 14,0 para 25,5 mil e Sumaré passou de 101,8 mil para 226,8 mil habitantes. O quadro crítico do saneamento nestes municípios se explica por este crescimento aceleradoao qual se associam as dificuldades para investimento público em uma década de severa crise econômica, a diversidade das prioridades políticas e o volume expressivo da demanda por serviços. (CARMO, 2001).

O segundo eixo do $\mathrm{PCO}$ pode ser interpretado como o potencial de atração de imigrantes, e não mostrou correlação com nenhuma das variáveis de qualidade ambiental. Por outro lado, este eixo pode ser usado para identificar pólos de atração na BPC, e propor diretrizes de gestão em função de seus indicadores de qualidade ambiental. Tais diretrizes poderiam ordenar melhor o processo de ocupação e crescimento destes pólos, reduzindo a degradação da qualidade ambiental observada nos pólos consolidados e adensados .

A Figura 4 mostra um exemplo. Com um gráfico do percentual de vegetação nativa dos municípios em função dos seus escores no segundo eixo do $\mathrm{PCO}$, é possível visualizar municípios que são pólos de atração (escores maiores que zero) e que ainda têm mais de $10 \%$ de sua área ocupada por vegetação nativa (Fig. 4, esses municípios estão assinalados no Apêndice). Em um planejamento regional, o zoneamento ambiental nesses municípios deve receber atenção prioritária, pois neles haverá, provavelmente, forte pressão de ocupação das áreas de vegetação nativa. Embora apenas pólos de atração com as maiores áreas de vegetação nativa tenham sido assinalados, a Figura 4 permite identificar grupos de municípios com qualquer combinação das variáveis demográficas e ambientais (e.g. pólos de atração com pouca vegetação, áreas de expulsão com muita vegetação) e definir, para cada um destes grupos, diretrizes de gestão apropriadas.

A Figura 5 mostra outro exemplo. Cruzando o segundo eixo da PCO com variáveis de saneamento ambiental, identificamos pólos de atração com cobertura de saneamento inferiores a 50\% dos domicílios (assinalados no Apêndice), e, portanto, com premência de ampliação da rede de água e esgoto para atender a uma população em expansão.

\section{CONCLUSÕES}

Esperamos ter demonstrado, ou pelo menos provocado, a utilidade que as ferramentas multivariadas têm para a pesquisa interdisciplinar, especialmente em questões ambientais. Acreditamos que o tratamento multivariado contribui, por vezes substancialmente, para elucidar aspectos ou sugerir processos que não seriam percebidos por tratamentos descritivos mais conservadores. Análises multivariadas prestam-se, por definição, à exploração de relações estruturais entre conjuntos de dados produzidos por disciplinas diferentes. Assim, são uma ferramenta essencial para detectar relações que, de outra forma, permaneceriam insuspeitas. O potencial exploratório da análise multivariada, tal como a empregamos aqui, é seu ponto mais forte. 
O alcance destas análises vai muito além do que experimentamos neste trabalho. Ativemo-nos ao PCA e ao PCO, mas há um vasto repertório de outras técnicas de ordenação e de classificação de grande utilidade para análise exploratória (GAUCH, 1982; DILLON \& GOLDSTEIN, 1984; BERNSTEIN et al., 1988; MANLY, 1994; JONGMAN et al., 1995). Além disso, é possível fazer testes formais de hipóteses, o que envolve diversos outros aspectos que aqui não foram tocados (ver, por exemplo, GREEN, 1979; SCHEINER \& GUREVITCH 1993; PRADO \& LEWINSOHN 1998).

Vale aqui uma comparação com a regressão linear (simples ou múltipla), uma das análises estatísticas mais utilizadas em ciências sociais (e.g., GORDON, 1968; KING et al., 1994). As técnicas de ordenação, aqui discutidas, são uma maneira de explorar os dados, expressando sua estrutura de correlações com o menor número possível de dimensões, sem perda importante de informação. Não há uma hipótese a ser testada a priori, e não é necessário postular causalidades, apenas correlações. Dependendo da técnica usada, não há sequer a exigência de relações lineares entre as variáveis. As regressões, ao contrário, testam hipóteses explícitas de que há efeitos aditivos (ou seja, lineares) de uma ou mais variáveis explanatórias sobre uma ou mais variáveis independentes. Sua principal vantagem é a validação estatística de modelos causais preditivos. A nosso ver, são técnicas complementares, que juntas podem compor uma estratégia de exploração de um conjunto complexo de variáveis, através de ordenação, para inferir hipóteses a sertestadas formalmente com regressões.

Os métodos experimentados neste trabalho sofreram muito pouca adaptação para serem empregados, o que não é por acaso. Sua ampla utilização na ecologia deve-se à capacidade de analisar sistemas complexos e conjuntos de dados com características, estrutura e precisão muito distintas. Além disto, como já indicamos na introdução, muitos destes métodos têm sido intercambiados com outras disciplinas, como a economia e a psicologia, que se aproximam bastante da ecologia quanto à heterogeneidade e disparidade de dados que freqüentemente precisam ser conciliados e integrados.

Como resultado da exploração multivariada interdisciplinar, surgem tanto hipóteses formais como indicações práticas, que podem ser usadas para interferir diretamente nos processos de planejamento e gestão. Assim, estas análises podem contribuir, em muito, para processos integrados de tomada de decisão. Entretanto, pela sua aparente complexidade e pelo aspecto formalmente exato de seus resultados, há aí um forte risco de que se mistifique a "objetividade" ou isenção das análises. Métodos multivariados são tanto, ou tão pouco, objetivos - no sentido de isenção ou imparcialidade - como outros procedimentos científicos. O modo de organizar os dados, e a estruturação e estratégia de análise, envolvem uma série de decisões que em geral irão afetar os resultados produzidos. Como dissemos de início, análises multivariadas são, em essência, métodos para simplificação e síntese de dados complexos. Assim, têm imenso potencial como ferramentas de apoio à tomada de decisões; mas não podem substituir procedimentos de informação e escolha por parte dos atores efetivos.

As relações que detectamos são estruturais, mas sua interpretação como processos decorrentes de relações causais dependem, por sua vez, do conhecimento 
disciplinar. $\mathrm{O}$ avanço na utilização de métodos multivariados na pesquisa interdisciplinar depende, em última instância, do envolvimento de todos os pesquisadores e de seu emprego em todas as fases do projeto. Esta é a única maneira de garantir o aprendizado recíproco que permitirá aprofundar progressivamente as questões que se propõem, e a densidade, extensão e utilidade das respostas que serão obtidas.

\section{BIBLIOGRAFIA}

BERNSTEIN, I.H.; TENG, G.K. \& GARBIN, C.P.. Applied multivariate analysis. New York, Spriger-Verlag, 1988.

BAENINIGER R. Espaços ganhadores e espaços perdedores na dinâmica migratória paulista. In: HOGAN, D.J.; CUNHA, J.M.P.; BAENINGER, R. \& CARMO, R.L. (org.). Migração e Ambiente em São Paulo - Aspectos relevantes da dinâmica recente. Campinas, NEPO/Unicamp. pp. 173-229, 2000.

BALTAR, V.T.; HOGAN, D.J. \& MORAN, R.C. Estudo das semelhanças e diferenças entre os municípios dos Rios Piracicaba, Capivari e Jundiaí. In: HOGAN, D.J.; CUNHA, J.M.P.; BAENINGER, R. \& CARMO, R.L. (org.). Migração e Ambiente em São Paulo - Aspectos relevantes da dinâmica recente. Campinas, NEPO/ Unicamp. pp. 413-438, 2000.

BOOKSTEIN, F.L. "Size and shape: a comment of semantics". Systematic Zoology 38 :173-180, 1989.

CARMO, R.L. A água é o limite? Redistribuição espacial da população e recursos hídricos no Estado de São Paulo. Campinas, IFCH/UNICAMP (Tese de Doutorado), 2001.

CUNHA, J.M.P.; BAENINIGER R.; CARMO, R.L. \& ANTICO, C. Dinâmica migratória no Estado de São Paulo. In: HOGAN, D.J.; CUNHA, J.M.P.; BAENINGER, R. \& CARMO, R.L. (org.). Migração e Ambiente em São Paulo - Aspectos relevantes da dinâmica recente. Campinas, NEPO/Unicamp. pp. 61-123, 2000.

DILLON, W.R. \& GOLDSTEIN, M. Multivariate analysis. Methods and applications. New York, John Wiley \& Sons, 1984.

GAUCH, H.G.J. Multivariate analysis in community ecology. Cambridge, Cambridge University Press. 1982.

GORDON, R.A. "Issues in multiple regression". American Journal of Sociology, 73 :592616, 1968.

GREEN, R.H. Sampling design and statistical methods for environmental biologists. New York, Wiley-Interscience, 1979.

HOGAN, D.J.; PEREZ-FILHO, A.; JANUZZI, G.M.;LEITÃO-FILHO, H.F. \& JOLY, C.A. Qualidade Ambiental e Desenvolvimento regional nas Bacias dos Rios Piracicaba e Capivari - Relatório Final. Campinas, NEPAM, 1997.

HOGAN, DJ.; CARMO, R.L.; RODRIGUES, I.A. \& ALVES, H. Crescimento Populacional e Migração nas Bacias dos Rios Piracicaba e Capivari: 1980. 1996. (Cadernos Qualidade Ambiental e Desenvolvimento regional nas Bacias dos Rios Piracicaba e Capivari, Vol. 1), Campinas, NEPAM, 1998. 
JONGMAN, R.H.G.; TER BRAAK, C.J.F. \& VAN TONGEREN, O.F.R. Data analysis in community and landscape ecology. 2nd ed.,Cambridge, Cambridge Univ. Press, 1995.

KING, G., KEOHANE, R.O. \& VERBA, S. Designing Social Inquiry - Scientific inference in qualitative research. Princeton, Princenton University Press, 1994.

KREBS, C.J. Ecological Methodology. New York, Harper \& Row, 1989.

MANLY, B.F.J. Multivariate statistical methods: a primer. 2nd ed., London, Chapman \& Hall, 1994.

MOSIMANN, J.E. \& JAMES, F.C. "New statistical method for allometry with application to Florida red-winged blackbirds". Evolution, 33 :444-459, 1979.

PIELOU, E.C. The interpretation of ecological data; a primer on classification and ordination. New York, Wiley, 1984.

PRADO, P.I.K.L. \& LEWINSOHN, T.M. O Uso de Análises Multivaridas Ecológicas em Estudos Ambientais Interdisciplinares. . (Cadernos Qualidade Ambiental e Desenvolvimento regional nas Bacias dos Rios Piracicaba e Capivari, Vol. 5), Campinas, NEPAM, 1998.

PERES-NETO, P.R. Introdução a análises morfométricas. In: PERES-NETO, P.R.; VALENTIN, J.L. \& FERNANDEZ, F.A.S. (orgs.) Tópicos em tratamentos de dados biológicos (série Oecologia Brasileinsis, Vol. II). Rio de Janeiro, PPGEUFRJ, 1995.

SCHEINER, S.M. \& GUREVITCH, J. (Eds.). Design and analysis of ecological experiments. New York, Chapman and Hall, 1993.

SECRETARIA DO MEIO AMBIENTE DO ESTADO DE SÃO PAUlO. Perfil Ambiental do Estado de São Paulo. (CD-ROM). São Paulo, SMA. 1999.

SNEATH, P.H.A. \& SOKAL, R.R. Numerical taxonomy. San Francisco, W.H. Freeman, 1973.

\section{NOTAS}

\footnotetext{
"Núcleo de Estudos e Pesquisas Ambientais (NEPAM), Campinas State University, Brazil.

** Núcleo de Estudos e Pesquisas Ambientais (NEPAM), Department of Zoology, Campinas State University, Brazil

*** Núcleo de Estudos de População (NEPO), Campinas State University, Brazil.

***** Núcleo de Estudos de População (NEPO), Campinas State University, Brazil.
}

\section{AGRADECIMENTOS}

Este trabalho é resultado do projeto interdisciplinar "Qualidade Ambiental e Desenvolvimento regional nas Bacias dos Rios Piracicaba e Capivari", executado pelo NEPAM-UNICAMP. Nossos agradecimentos à sua equipe de pesquisadores, de técnicos e de apoio, que de várias maneiras auxiliaram esta pesquisa. Nossos agradecimentos à FAPESP e ao FINEP - PADCT/CIAMB, pelo apoio financeiro ao projeto. Um assessor anônimo contribuiu decisivamente para o aperfeiçoamento do texto originalmente apresentado. Este artigo é dedicado à memória de Maria Lúcia Leonardi e Hermógenes F. Leitão-Filho, nossos colegas pesquisadores no projeto. 
Tabela 1 - Autovetores dos dois primeiros eixos do PCO: vetores dos coeficientes das variáveis demográficas em cada eixo. O primeiro eixo tem um autovalor de 2,123 e explica 53\% da variação dos dados. O segundo eixo tem um autovalor de 0,945, e explica os outros $24 \%$ da variação.

\begin{tabular}{|l|r|r|}
\hline Variável & Eixo 1 & Eixo 2 \\
\hline População residente & 0,550 & $-0,349$ \\
\hline \% Urbanização & 0,522 & $-0,418$ \\
\hline \% Crescimento Vegetativo & 0,545 & 0,221 \\
\hline \% Saldo migratório & 0,358 & 0,809 \\
\hline
\end{tabular}

Figura 1 - Escores dos municípios nos dois primeiros eixos do PCO. Legenda no Apêndice, autovalores dos eixos na Tabela 1. Municípios com escores elevados no primeiro eixo possuem as maiores populações residentes, taxas de urbanização, de crescimento vegetativo (e.g., 9 - Campinas, 45 - Sumaré, 40 - Santa Bárbara do Oeste). Municípios com escores elevados no segundo eixo possuem saldos migratórios elevados, independente de seus tamanhos populacionais e taxa de urbanização (e.g. 45 - Sumaré, 5 Artur Nogueira, 21 - Jarinu, 27 - Monte-Mor).

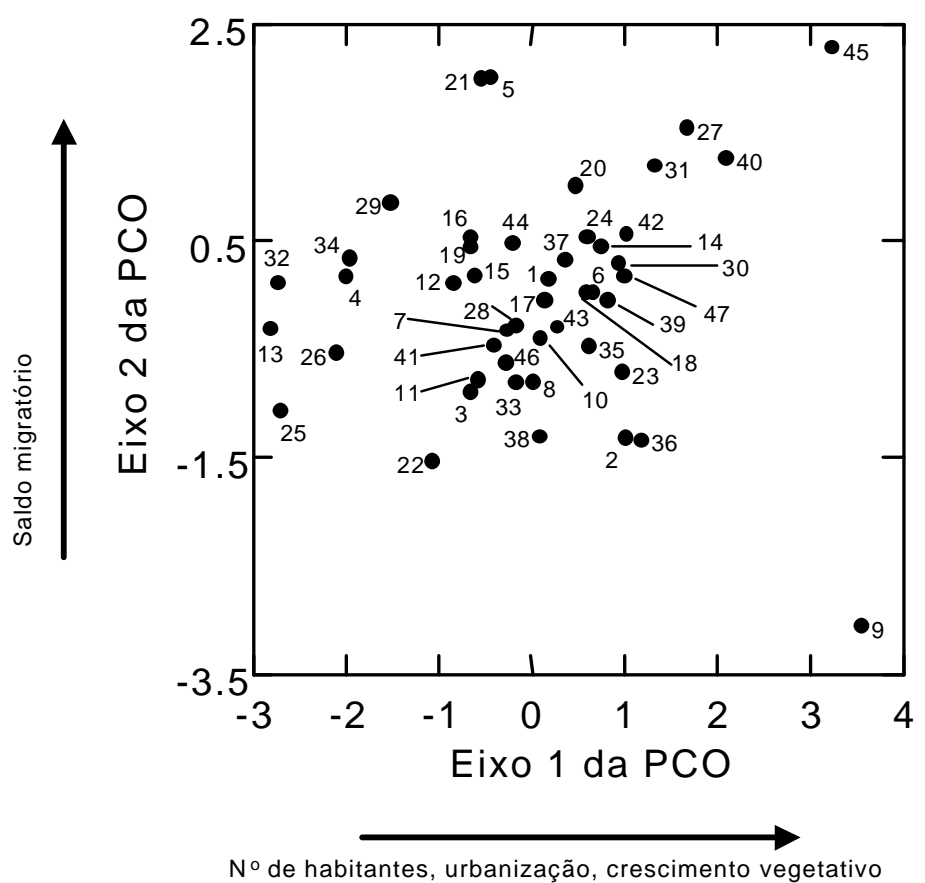


Figura 2 - Percentual de área dos municípios com mata nativa (1988) em função de suas coordenadas no primeiro eixo do PCO. Municípios com escores baixos no primeiro eixo (e.g., 4 - Analândia, 13 - Corumbataí, 25 - Mombuca, 26 - Monte Alegre do Sul, 32 Pedra Bela) tendem a ter maior percentual de sua área coberta com vegetação nativa, em comparação com os municípios com escores elevados (e.g., 9 - Campinas, 40 - Santa Bárbara do Oeste, 45 - Sumaré). Apesar da grande variação de cobertura vegetal em municípios com escores intermediários (e.g., 39 - Rio das Pedras vs. 35 - Piracaia), o coeficiente de correlação de Spearman é significativo $(r=-0,469 ; \mathrm{p}=0,01)$, indicando uma tendência à redução da área de vegetação nativa com o aumento da população e urbanização. Abcissa em escala logarítmica, para melhor visualização.

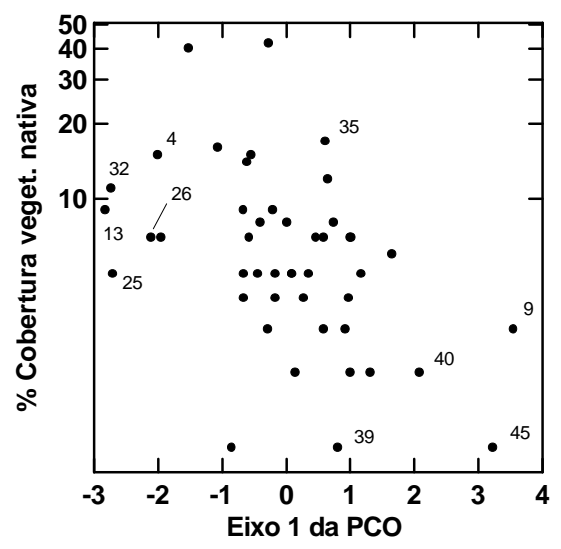

Figura 3 - Percentual dos domicílios ligados à rede de esgoto em cada município (1991) em função de suas coordenadas no primeiro eixo do PCO. Há uma correlação significativa $(\mathrm{r}=0,513 ; \mathrm{p}=0,0002)$, embora alguns municípios, assinalados com números (6 - Atibaia, 21 - Jarinu, 27 - Monte-Mor, 45 - Sumaré; ver apêndice), possuam um menor percentual de domicílios ligados à rede do que o esperado pelo tamanho, crescimento e grau de urbanização de suas populações.

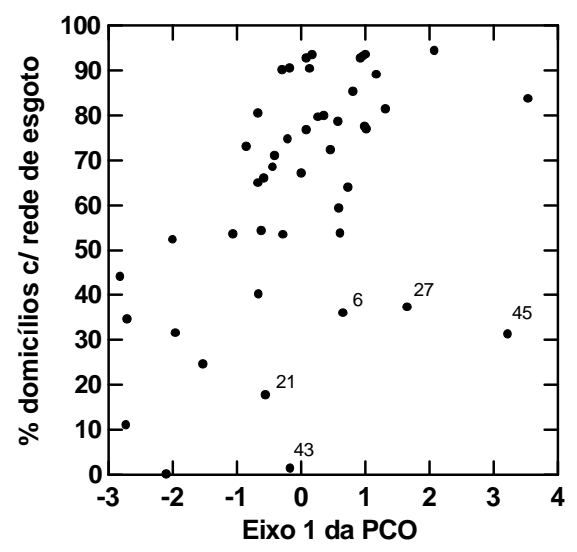


Figura 4 - Percentual de área dos municípios com mata nativa (1988) em função de suas coordenadas no segundo eixo do PCO. Os municípios em destaque são prioritários para medidas de planejamento de ocupação do solo, pois são pólos de atração populacional (escores elevados no segundo eixo do PCO) com mais de $10 \%$ de sua área rural coberta por mata nativa em 1985 (indicados com "+" no apêndice). Abcissa em escala logarítmica, para melhor visualização.

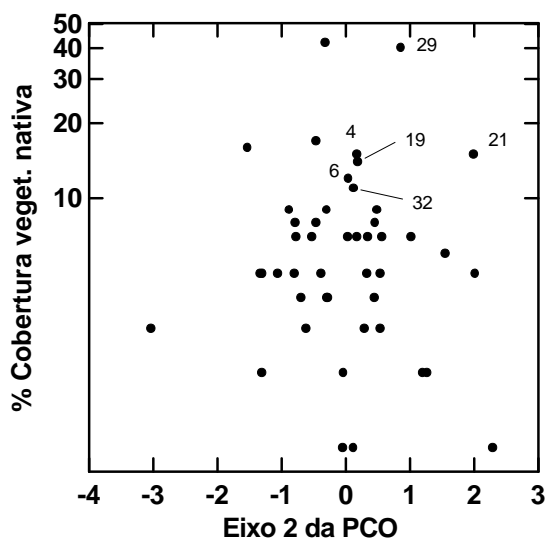

Figura 5 - Percentual dos domicílios ligados à rede de esgoto em cada município (1991) em função de suas coordenadas no segundo eixo do PCO. Municípios com escores elevados no segundo eixo do PCO e menos do que 50\% dos domićlios ligados à rede de esgoto estão assinalados como merecedores de atenção quanto ao saneamento, pois são pólos de atração populacional com rede de esgoto restrita (indicado com “*” no apêndice).

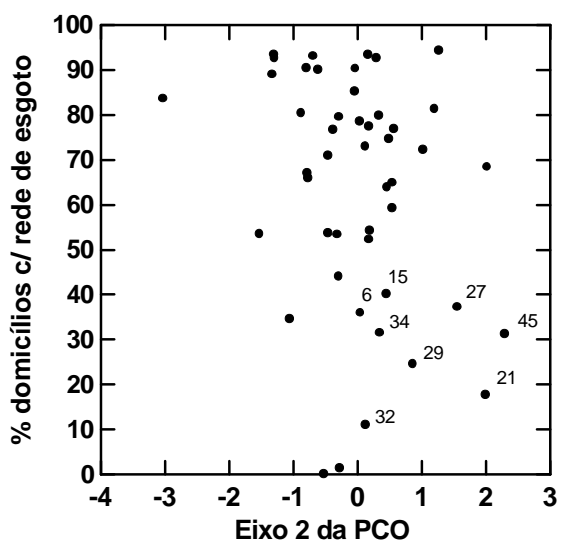


Apêndice- Municípios analisados, suas variáveis demográficas e ambientais, e suas coordenadas ("escores") nos dois primeiros eixos da análise de coordenadas principais (PCO). Os códigos identificam os municípios no diagrama (Figura 1). Municípios prioritários para planejamento de ocupação do solo estão indicados com "+", e os prioritários para expansão da rede de esgoto estão indicados com “*” (Ver Figuras 4 e 5 e texto para explicações).

\begin{tabular}{|c|l|r|r|r|r|r|r|r|r|}
\hline Cód. & Município & Pop. & \% Urban & \%Cveg & \%Smigr & \%Veget & \%sgot & Eixo1 & Eixo2 \\
\hline 1 & Águas de São Pedro & 1697 & $100.00^{2}$ & 18.39 & 36.69 & 0 & 93.43 & 0.173 & 0.161 \\
\hline 2 & Americana & 153840 & 99.88 & 25.47 & 0.57 & 2 & 93.62 & 1.006 & -1.307 \\
\hline 3 & Amparo & 50797 & 81.54 & 18.56 & 3.11 & 9 & 80.50 & -0.67 & -0.883 \\
\hline 4 & Analândia + & 3020 & 52.72 & 13.12 & 18.08 & 15 & 52.34 & -2.003 & 0.175 \\
\hline 5 & Artur Nogueira & 28053 & 48.42 & 21.07 & 54.86 & 5 & 68.52 & -0.442 & 2.016 \\
\hline 6 & Atibaia + * & 86336 & 86.58 & 24.24 & 24.86 & 12 & 35.97 & 0.654 & 0.039 \\
\hline 7 & Bom Jesus dos Perdóes & 9854 & 91.29 & 18.69 & 19.94 & 42 & 53.43 & -0.276 & -0.317 \\
\hline 8 & Bragança Paulista & 108980 & 84.79 & 20.80 & 8.41 & 8 & 67.17 & 0.011 & -0.789 \\
\hline 9 & Campinas & 847595 & 97.33 & 22.73 & 4.64 & 3 & 83.73 & 3.545 & -3.038 \\
\hline 10 & Capivari & 34220 & 84.29 & 25.46 & 10.30 & 4 & 79.65 & 0.271 & -0.287 \\
\hline 11 & Charqueada & 10735 & 80.80 & 21.46 & -0.76 & 7 & 66.03 & -0.58 & -0.775 \\
\hline 12 & Cordeirópolis & 13338 & 75.69 & 16.28 & 25.79 & 1 & 73.05 & -0.853 & 0.116 \\
\hline 13 & Corumbataí & 3156 & 40.21 & 11.44 & 1.29 & 9 & 44.02 & -2.82 & -0.299 \\
\hline 14 & Cosmópolis & 36684 & 89.42 & 24.87 & 32.96 & 8 & 64.03 & 0.737 & 0.456 \\
\hline 15 & Elias Fausto * & 11632 & 59.43 & 22.82 & 17.48 & 4 & 40.21 & -0.662 & 0.45 \\
\hline 16 & Ipeúna & 2698 & 65.94 & 20.44 & 25.53 & 5 & 64.96 & -0.668 & 0.537 \\
\hline 17 & Iracemápolis & 11752 & 89.87 & 22.14 & 22.20 & 2 & 90.45 & 0.14 & -0.039 \\
\hline 18 & Itatiba & 61645 & 87.72 & 24.45 & 23.49 & 7 & 78.57 & 0.583 & 0.036 \\
\hline 19 & Itirapina + & 9953 & 75.47 & 18.69 & 24.41 & 14 & 54.36 & -0.613 & 0.19 \\
\hline 20 & Jaguariúna & 24999 & 76.35 & 24.87 & 39.51 & 7 & 72.28 & 0.46 & 1.017 \\
\hline 21 & Jarinu +* & 10878 & 50.18 & 20.31 & 55.11 & 15 & 17.73 & -0.551 & 1.997 \\
\hline 22 & Joanópolis & 8186 & 87.45 & 17.62 & -12.04 & 16 & 53.50 & -1.063 & -1.532 \\
\hline 23 & Limeira & 207770 & 85.64 & 24.64 & 13.13 & 4 & 93.21 & 0.973 & -0.698 \\
\hline 24 & Louveira & 16259 & 86.91 & 24.95 & 32.38 & 3 & 59.33 & 0.588 & 0.543 \\
\hline 25 & Mombuca & 49.02 & 12.87 & -15.17 & 5 & 34.57 & -2.707 & -1.058 \\
\hline
\end{tabular}




\begin{tabular}{|c|c|c|c|c|c|c|c|c|c|}
\hline Cód. & Município & Pop. & \%Urban & \%Cveg & \%Smigr & \%Veget & \%Esgot & Eixo1 & Eixo2 \\
\hline 26 & Monte Alegre do Sul & 5439 & 50.56 & 15.95 & -4.22 & 7 & 0.00 & -2.104 & -0.523 \\
\hline 27 & Monte Mor * & 25559 & 86.25 & 31.13 & 50.86 & 6 & 37.30 & 1.656 & 1.55 \\
\hline 28 & Morungaba & 8210 & 76.11 & 23.66 & 2.01 & 8 & 71.02 & -0.406 & -0.457 \\
\hline 29 & Nazaré Paulista $+*$ & 11671 & 35.66 & 20.75 & 17.67 & 40 & 24.56 & -1.525 & 0.859 \\
\hline 30 & Nova Odessa & 34063 & 93.86 & 26.07 & 29.68 & 3 & 92.78 & 0.928 & 0.298 \\
\hline 31 & Paulínia & 36706 & 89.65 & 27.38 & 48.98 & 2 & 81.37 & 1.317 & 1.197 \\
\hline 32 & Pedra Bela $+*$ & 5142 & 17.25 & 18.76 & -9.08 & 11 & 10.98 & -2.732 & 0.123 \\
\hline 33 & Pedreira & 27972 & 95.82 & 19.31 & 10.50 & 5 & 90.57 & -0.173 & -0.799 \\
\hline 34 & Pinhalzinho $*$ & 8433 & 45.01 & 15.38 & 15.87 & 7 & 31.52 & -1.956 & 0.344 \\
\hline 35 & Piracaia & 18999 & $100.00^{\mathrm{a}}$ & 24.82 & 14.05 & 17 & 53.80 & 0.609 & -0.462 \\
\hline 36 & Piracicaba & 283833 & 95.11 & 21.96 & 10.40 & 5 & 89.13 & 1.172 & -1.33 \\
\hline 37 & Rafard & 8588 & 76.29 & 27.83 & 17.20 & 5 & 79.94 & 0.353 & 0.331 \\
\hline 38 & Rio Claro & 138243 & 96.02 & 18.16 & 6.58 & 5 & 92.81 & 0.083 & -1.299 \\
\hline 39 & Rio das Pedras & 19104 & 87.33 & 29.66 & 11.97 & 1 & 85.37 & 0.81 & -0.046 \\
\hline 40 & Santa Bárbara d'Oeste & 145266 & 97.19 & 26.28 & 63.38 & 2 & 94.44 & 2.081 & 1.265 \\
\hline 41 & Santa Gertrudes & 10485 & 92.46 & 19.47 & 11.89 & 3 & 90.14 & -0.291 & -0.621 \\
\hline 42 & Santa Maria da Serra & 4303 & 80.55 & 32.29 & 19.76 & 7 & 77.02 & 1.017 & 0.565 \\
\hline 43 & Santo Antonio de Posse & 14327 & 78.39 & 24.11 & 7.69 & 4 & 1.38 & -0.171 & -0.278 \\
\hline 44 & São Pedro & 20176 & 77.62 & 20.20 & 31.92 & 9 & 74.73 & -0.208 & 0.484 \\
\hline 45 & Sumaré * & 226870 & 99.41 & 28.14 & 94.01 & 1 & 31.28 & 3.224 & 2.291 \\
\hline 46 & Valinhos & 67886 & 88.25 & 20.71 & 18.01 & 5 & 76.80 & 0.088 & -0.383 \\
\hline 47 & Vinhedo & 33612 & 98.18 & 25.72 & 29.41 & 7 & 77.49 & 0.993 & 0.181 \\
\hline
\end{tabular}

a - Nota metodológica: é importante salientar que o IBGE respeita na captação e divulgação de seus dados as delimitações de áreas rurais e urbanas que são estabelecidas através da legislação municipal. Alguns municípios, em função de possíveis aumentos na arrecadação de impostos, estabelecem que a totalidade de seu território configura em áreas urbanas. Isto explica o elevado percentual de população urbana registrado para municípios pequenos, como Águas de São Pedro e Piracaia. 


\section{Rich nature, poor people? Conceptual and analytical questions on the role of natural resources for contemporary prosperity}

This article questions the current trend which supposes that the rich natural resource endowment of a region leads to the prosperity or weel-being of its inhabitants. It discusses classic statements by Prebisch and Hirschman as well as more contemporary ones by Bunker and Freudenburg. These authors stress the idea that regions and activities intensive in natural resources have serious developmental handicaps when compared to regions and activities intensive in industrial transformation of natural resources or in technology and information.

Key words: natural resources / development / extractive economies / transformative industries / added value / raw products

\section{PAULO InÁCIO \\ THOMAS LEWINSOHN \\ ROBERTO LUIZ DO CARMO \\ Daniel Joseph Hogan}

\section{Ordenação multivariada na ecologia e seu uso em ciências ambientais}

Análises multivariadas rotineiras em ecologia podem ser facilmente aplicadas em outras ciências ambientais, oferecendo novas possibilidades de exploração analítica e quantitativa de padrões complexos. Demonstramos isto com um estudo das relações entre variáveis demográficas e de qualidade ambiental nas Bacias dos Rios Piracicaba e Capivari. Vetores demográficos foram identificados com uma análise de coordenadas principais e, em seguida, correlacionados com variáveis de saneamento e de cobertura vegetal. A análise evidenciou a estrutura de correlações entre as variáveis, indicando tanto hipóteses formais sobre as suas causas, como diretrizes práticas de gestão. Análises multivariadas são muito eficazes para a exploração de relações estruturais entre uma grande quantidade de variáveis, sendo uma ferramenta importante em estudos ambientais interdisciplinares.

Palavras-chave: análises multivariadas, ecologia, ciências ambientais.

\section{Multivariate ordering in ecology and its use for environmental sciences}

Multivariate analyses usual to ecology can be easily applied in other environmental sciences, making available new analytical tools for the quantitative exploration of complex patterns. We exemplify this through a study of the relations among demographic variables and environmental quality in the Piracicaba and Capivari River Basins. Demographic vectors were found by a principal coordinates analysis, and then were correlated with variables of sanitation and of vegetation cover. This analysis revealed the structure of correlations among the variables, suggesting causative hypotheses, as well as practical guidelines in environmental management. Multivariate analyses are very effective in exploring structural links among 
many variables, which makes them an important tool in interdisciplinary studies on environment.

Keywords: multivariate analyses, ecology, environmental sciences.

$$
\text { SÉRGIO Luís BOEIRA }
$$

Political Ecology: GuerreiroRamos and Fritjof Capra

The aim of this essay is to contribute with the articulation of a trans-disciplinary research field, political ecology, by means of the comparative synthesis of two works: "The New Science of Organizations" by Alberto Guerreiro Ramos (1981), and "The Mutation Point", by Fritjof Capra (1982). The central hypothesis of this article is that both authors, although with very different academic background, get to very similar conclusions in these two works, under the same emergent paradigm. Given the still diffuse context of environmentalist thought these books may be considered classics of political ecology.

Keywords: paradigm, ecology, instrumental reason, mechanistic, systemic.

\title{
Ecologia Política: Guerreiro Ramos e Fritjof Capra
}

Este ensaio tem por objetivo contribuir com a articulação de um campo de pesquisa transdisciplinar, a ecologia política, por meio da síntese comparativa de duas obras: A Nova Ciência das Organizações, de Alberto Guerreiro Ramos (1981), e O Ponto de Mutação, de Fritjof Capra (1982). A hipótese central deste trabalho é que os autores, apesar de suas formações acadêmicas muito diferentes, chegaram a resultados muito semelhantes nestas obras, a partir de um mesmo paradigma emergente. Dado o contexto ainda difuso do pensamento ambientalista, estes livros podem ser considerados clássicos da ecologia política. Palavras-chave: paradigma, ecologia, razão instrumental, mecanicismo, sistêmico.

\author{
CÉlia FutTEMA \\ FÁBIO DE CASTRO \\ Maria Clara Silva-Forsberg \\ ELINOR OSTROM
}

\section{A Emergência e os Resultados da Ação Coletiva: Uma abordagem institucional e ecossistêmica}

A participação numa ação coletiva é estudada com freqüência por meio de uma análise baseada nas comunidades e enfocada nas características sociais dos participantes e nas características ecológicas do sistema manejado. Este estudo refere-se à importância de se limitar a análise ao nível das famílias para compreender os diferentes incentivos 